\title{
Area postrema syndrome
}

\section{Frequency, criteria, and severity in AQP4-IgG-positive NMOSD}

Eslam Shosha, MD, Divyanshu Dubey, MD, Jacqueline Palace, FRCP, DM, Ichiro Nakashima, MD, PhD, Anu Jacob, MD, FRCP, Kazuo Fujihara, MD, PhD, Toshiyuki Takahashi, MD, Daniel Whittam, MBChB, Maria Isabel Leite, MD, DPhil, Tatsuro Misu, MD, PhD, Takai Yoshiki, MD, PhD, Silvia Messina, MD, Liene Elsone, MD, Masoud Majed, MD, Eoin Flanagan, MD, Avi Gadoth, MD, Carey Huebert, BS, Jessica Sagen, BA, Benjamin M. Greenberg, MD, Michael Levy, MD, PhD, Aditya Banerjee, BS, Brian Weinshenker, MD, and Sean J. Pittock, MD

Neurology ${ }^{\circledR}$ 2018;91:e1642-e1651. doi:10.1212/WNL.0000000000006392

\section{Abstract}

\section{Objective}

To define the frequency, duration, and severity of intractable nausea, vomiting, or hiccups in aquaporin-4-immunoglobulin G (AQP4-IgG)-positive neuromyelitis optica spectrum disorder (NMOSD) and propose diagnostic criteria and a severity scale for area postrema syndrome (APS).

\section{Methods}

An International NMOSD database was interrogated for frequency of APS. Patients with AQP4-IgG-positive NMOSD completed an APS symptom questionnaire. Nausea and vomiting severity was derived from the Pregnancy-Unique Quantification of Emesis and Nausea (PUQE) score. The diagnostic criteria, severity scale, and immunotherapy response was applied to a prospective validation cohort of patients from multiple centers.

\section{Results}

Analysis of an international database for AQP4-IgG-seropositive NMOSD $(n=430)$ revealed a high prevalence of isolated APS attacks (onset 7.1\%-10.3\%; subsequent 9.4\%-14.5\%) across continents. For 100 patients with 157 episodes of APS, nausea ( $n=127,81 \%)$ lasted for a median of 14 days (range $2-365)$, vomiting $(113,72 \%)$ with a median of 5 episodes/d (2-40) lasted 1-20 minutes, and hiccups (102, 65\%) lasted a median of 14 days (2-365). Symptoms consistently and completely resolved following immunotherapy. Data were used to propose APS diagnostic criteria and repurpose PUQE score (hiccups severity grade based on symptom duration). The clinical utility was demonstrated in a prospective validation cohort.

\section{Conclusion}

Isolated APS attacks are frequently encountered both at onset and during the NMOSD course. The diagnostic criteria proposed here will assist clinicians in recognizing APS. Diagnosis of an APS attack earlier than 48 hours is possible if a dorsal medulla lesion is detected. Accurate diagnosis and evaluation of APS attack severity will assist in outcome measurement in NMOSD clinical trials.

\author{
Correspondence \\ Dr. Pittock \\ pittock.sean@mayo.edu
}

From the Departments of Neurology (E.S., D.D., M.M., E.F., A.G., B.W., S.J.P.), Laboratory Medicine and Pathology (M.M., E.F., A.G., S.J.P.), and Clinical Research Unit (C.H., J.S.), Mayo Clinic College of Medicine, Rochester, MN; Nuffield Department of Clinical Neurosciences (J.P., M.I.L., S.M.), Oxford; The Walton Centre (A.J., D.W., L.E.), NHS Foundation Trust, Liverpool, UK; Department of Neurology (I.N., K.F., T.T., T.M., Y.T.), Tohoku University Graduate School of Medicine, Sendai; Department of Multiple Sclerosis Therapeutics (K.F.) Fukushima Medical University School of Medicine and Multiple Sclerosis and Neuromyelitis Optica Center, Southern Tohoku Research Institute for NeuroScience, Koriyama, Japan; Department of Neurology (M.L., A.B.), Johns Hopkins University, Baltimore, MD; Departments of Neurology and Neurotherapeutics (B.M.G.), UT Southwestern Medical Center, Dallas, TX; Department of Neurology (T.T.), Yonezawa National Hospital; and Department of Neurology (I.N.), Tohoku Medical and Pharmaceutical University, Sendai, Japan.

Go to Neurology.org/N for full disclosures. Funding information and disclosures deemed relevant by the authors, if any, are provided at the end of the article. 


\section{Glossary}

$\mathbf{A P}=$ area postrema; $\mathbf{A P S}=$ area postrema syndrome; $\mathbf{A Q P 4}=$ aquaporin-4; $\mathbf{H G}=$ hyperemesis gravidarum; IgG = immunoglobulin G; INVH = intractable nausea, vomiting, or hiccups; NMOSD = neuromyelitis optica spectrum disorder; ON = optic neuritis; PAGI-SYM = Patient Assessment of Upper Gastrointestinal Disorders-Symptoms Severity Index; PUQE = Pregnancy-Unique Quantification of Emesis and Nausea; TM = transverse myelitis.

Neuromyelitis optica spectrum disorders (NMOSD) are a clinically and radiologically defined group of CNS inflammatory autoimmune demyelinating diseases, the majority of which are associated with a pathogenic antibody specific for the aquaporin-4 (AQP4) water channel. ${ }^{1-3}$ NMOSD-typical brain MRI lesions occur in AQP4-enriched areas ${ }^{4,5}$ including the area postrema (AP), an emetic reflex center ${ }^{6}$ at the floor of the fourth ventricle that is penetrated by convoluted capillaries that lack tight endothelial junctions. ${ }^{6,7}$ The AP, via hypothalamic/brainstem connections, regulates fluid balance, osmoregulation, immunomodulation, and other important physiologic systems, ${ }^{8}$ and also has chemo-sensitive neurons mediating hiccups. ${ }^{9}$

NMOSD lesions in the AP are associated with loss of AQP4 immunoreactivity and with inflammation, but different from spinal and optic lesions, lack demyelination and necrosis. This difference may explain the almost universal complete remission of symptoms from such lesions. ${ }^{5,10,11}$ Experimental ablation arrests intractable vomiting, ${ }^{12}$ and an increase in neuronal firing is associated with projectile vomiting. ${ }^{13}$ Attacks of intractable nausea, vomiting, or hiccups (INVH), in the context of a lesion in the dorsal medulla, are known as AP syndrome (APS). ${ }^{14}$ Up to $30 \%$ of patients will have an APS during their illness. ${ }^{5,14-16}$

APS is included as a core clinical criterion for NMOSD; however, the APS phenotype (onset, frequency, duration, severity, associated symptoms, and treatment response) is not well described. Herein, we define the frequency, duration, and severity of INVH in AQP4-IgG-positive NMOSD and propose diagnostic criteria and a severity scale for APS.

\section{Methods}

\section{Standard protocol approvals, registrations, and patient consents}

All patients in our study consented to the use of their medical record for research purposes. All patients responded to a telephone questionnaire and provided informed written consent. The study was approved by each center's ethics committee as follows. In the United Kingdom: National Research Ethics Service (London-Hampstead) REC reference 15/LO/1433, Project ID 180720; Oxford Research Ethics Committee C Ref: 10/H0606/56. In Japan: ethical committee of Tohoku University School of Medicine (no. 2013-130). In the United States: institutional review board of Mayo Clinic, Rochester, MN (IRB no. 08-007846).
Three separate NMOSD patient cohorts (all AQP4IgG-positive by cell-based assays) were evaluated for this study as follows.

\section{Aim 1: To report the frequency of isolated APS attacks across continents and in different ethnicities, by analyzing a large international NMOSD attack database}

An international database defining dates and types of NMOSD attacks in 430 patients from the United Kingdom, Japan, and the United States was interrogated.

\section{Aim 2: To further characterize the APS phenotype and propose guidelines for APS diagnosis and investigate the utility of a repurposed nausea and vomiting in pregnancy severity scale (Pregnancy-Unique Quantification of Emesis and Nausea) with addition of a hiccups component for NMOSD}

From the Mayo Clinic NMOSD database, 100 patients (none from the international database) with a total of 157 APScompatible symptoms otherwise unexplained (e.g., medication side effects, anorexia nervosa, gastrointestinal disorder, pregnancy, or other comorbid condition) were interviewed. Clinical information collected during telephone interview included symptom type and onset, frequency, duration, associated symptoms (e.g., weight loss, yawning, menstrual irregularity, and headache), severity, timing of other NMOSD core attacks (e.g., optic neuritis $[\mathrm{ON}]$, transverse myelitis $[\mathrm{TM}]$, brainstem), and symptomatic (antiemetic medications) and immunotherapy treatment responses. We documented any initial gastroenterology diagnosis from patients' records. All available brain MRIs at the time of APS were reviewed. Utilizing these data, we defined clinical criteria for APS diagnosis in AQP4-IgG-seropositive NMOSD.

The severity scale for APS was modified from a validated score: the Pregnancy-Unique Quantification of Emesis and Nausea (PUQE) score (table 1). Nausea and vomiting severity was based on duration of nausea and frequency of vomiting episodes in accordance with validated PUQE criteria, demonstrated to be correlated with quality-of-life measures over a 24-hour period of symptoms. Since hiccups are not included in the PUQE criteria, a separate hiccups section based on duration of symptoms was added.

\section{Aim 3: To validate the revised NMOSD PUQE scale in a prospective multicenter cohort of patients with NMOSD}

These criteria and the severity scale were applied to a different subgroup of 25 patients with NMOSD who had recent APS 
Table 1 PUQE form

Pregnancy-unique quantification of emesis and nausea

\begin{tabular}{|c|c|c|c|c|}
\hline \multicolumn{5}{|c|}{ 1. On average in a day, for how long do you feel nauseated or sick to your stomach? } \\
\hline$>6 \mathrm{~h}$ & $4-6 \mathrm{~h}$ & $2-3 \mathrm{~h}$ & $\leq 1 \mathrm{~h}$ & Not at all \\
\hline 5 points & 4 points & 3 points & 2 points & 1 point \\
\hline \multicolumn{5}{|c|}{ 2. On average in a day, how many times do you vomit or throw up? } \\
\hline$\geq 7 \mathrm{~h}$ & $5-6$ times & 3-4 times & $1-2$ times & Not at all \\
\hline 5 points & 4 points & 3 points & 2 points & 1 point \\
\hline \multicolumn{5}{|c|}{ 3. On average in a day, how many times have you had retching or dry heaves without bringing anything up? } \\
\hline$\geq 7 \mathrm{~h}$ & 5-6 times & 3-4 times & $1-2$ times & Not at all \\
\hline 5 points & 4 points & 3 points & 2 points & 1 point \\
\hline
\end{tabular}

Abbreviation: PUQE = Pregnancy-Unique Quantification of Emesis and Nausea.

Total score (sum of replies to 1, 2, and 3): mild nausea and vomiting in pregnancy (NVP) $\leq 6$; moderate NVP 7-12; severe NVP $\geq 13$. Quality of life question: On a scale of $0-10$, how would you rate your well-being? 0 = worst possible; 10 = as good as you felt before pregnancy. PUQE form modified from Koren et al., Am J Obstet Gynecol, with permission. ${ }^{23}$

attacks identified by coinvestigators at multiple international centers. The utility of the severity scale applied at APS nadir, post symptomatic therapy, and post immunotherapy was evaluated.

\section{Statistical analysis}

Univariate and post hoc analysis of continuous variables between antibody subgroups was performed using 1-way anal$y$ sis of variance. Nominal variables were analyzed using the $\chi^{2}$ test (IBM SPSS 23; IBM Corp., Armonk, NY). Because of the retrospective and exploratory nature of the study, no adjustment for multiple comparisons was made.

\section{Data availability statement}

The dataset used and analyzed during the current study is available from the corresponding author on reasonable request.

\section{Results}

\section{APS attacks (isolated or accompanying other symptoms) in patients with AQP4- IgG-seropositive NMOSD are common and occur with similar frequency across continents and ethnicities}

A total of 430 patients (59 males, 371 females) were evaluated from 3 different countries (Japan, $n=69$; United Kingdom, $n=$ 169; United States, $\mathrm{n}=192$ ) (figure $1, \mathrm{~A}-\mathrm{F}$ ). Age at symptom onset in patients from Japan (median age 40 years [range 13-73 years]), the United Kingdom (median age 47 [range 3-79 years]), and the United States (median age 43 years [range $5-76$ years $])$ was comparable $(p=0.680)$. The frequency of isolated APS at NMOSD onset was 7.1\% in UK, 8.7\% in Japanese, and $10.3 \%$ in US patients with NMOSD $(p=0.163)$. The frequency of APS occurring in association ( $<30$ days in between) with other symptoms (e.g., ON or TM) at onset attack was $11.2 \%$ in UK, $15.9 \%$ in Japanese, and $8.2 \%$ in US patients ( $p$ $=0.332$ ). APS attacks also occurred frequently during the course (not onset attack) of NMOSD; isolated APS occurred as noninaugural attacks in $11.2 \%$ in UK, $14.5 \%$ in Japanese, and $9.4 \%$ in US patients $(p=0.331)$ and in association with other symptoms in $12.4 \%$ in UK, $18.8 \%$ in Japanese, and $8.9 \%$ in US patients $(p=0.043)$. The frequency of APS attacks at onset and during the course of NMOSD stratified according to ethnicity across 3 continents is illustrated in figure 1 .

\section{The APS attack phenotype}

Of 100 patients (91 of whom were women), 45 Caucasian, 37 African American, 8 Asian, 7 Hispanic, and 3 others experienced 157 APS attacks (table 2). APS attacks were the inaugural symptoms in 54 patients. Of 157 APS attacks, 64 (40\%) occurred without other neurologic symptoms and 91 (58\%) preceded another NMO-typical relapse (19 ON, $58 \mathrm{TM}, 9 \mathrm{ON} /$ TM, 4 brainstem, 1 cerebral) by a median of 10 days (0-90). In only 2 patients did the APS symptoms follow an NMO-typical relapse (2 TM). Brain MRIs performed within 21 days (2-90) of APS onset revealed increased T2/fluid-attenuated inversion recovery or abnormal enhancement involving the dorsomedial medulla including the AP (figure 2).

\section{APS is frequently evaluated by gastroenterologists and misdiagnosis is common}

Forty-four patients presented initially to gastroenterologists and underwent extensive workup including upper gastrointestinal endoscopy, transit studies, and abdominal CT. The cause was not identified by the gastroenterologist in any of these patients. Twenty percent were given an incorrect diagnosis for their APS: gastroparesis (4), food poisoning (2), gastroesophageal reflux disease (2), Helicobacter pylori infection (2), cholecystitis (2), Crohn disease (1), pancreatitis 
Figure 1 Frequency of APS in patients with NMOSD stratified according to ethnicity

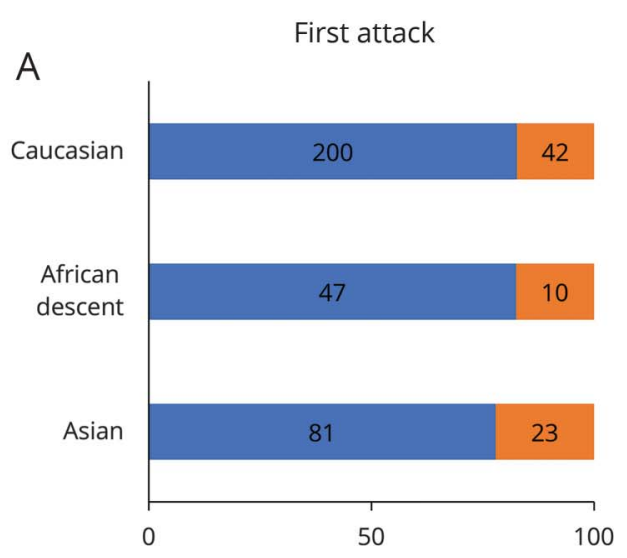

B

Subsequent attacks
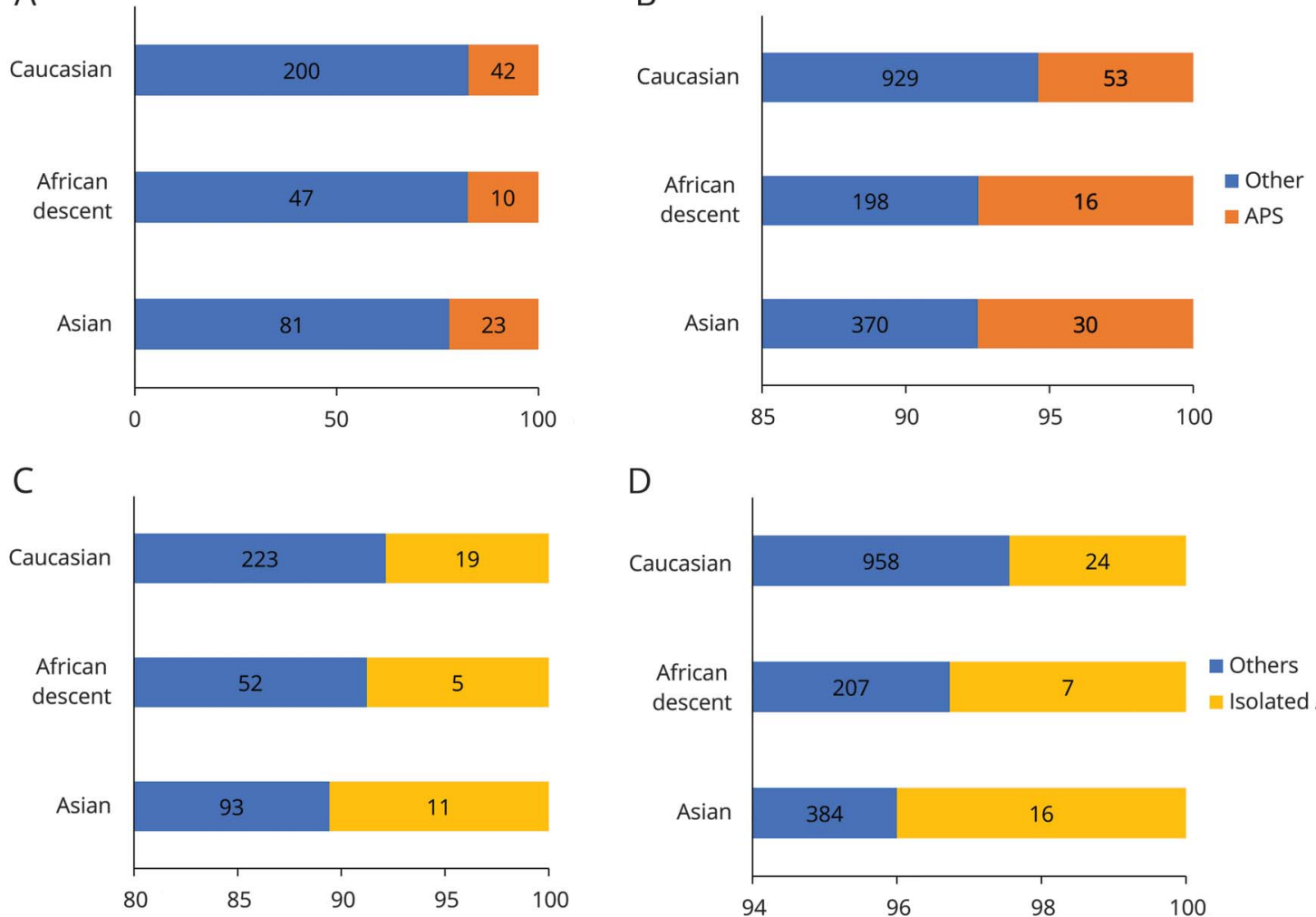

E

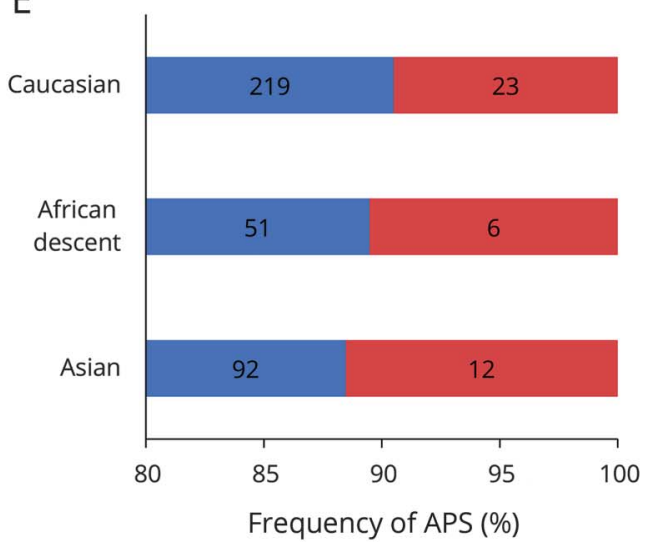

$\mathrm{D}$

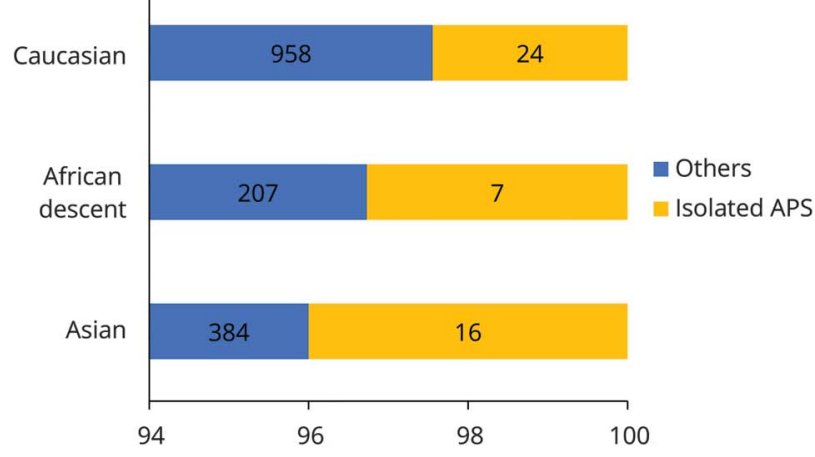

F

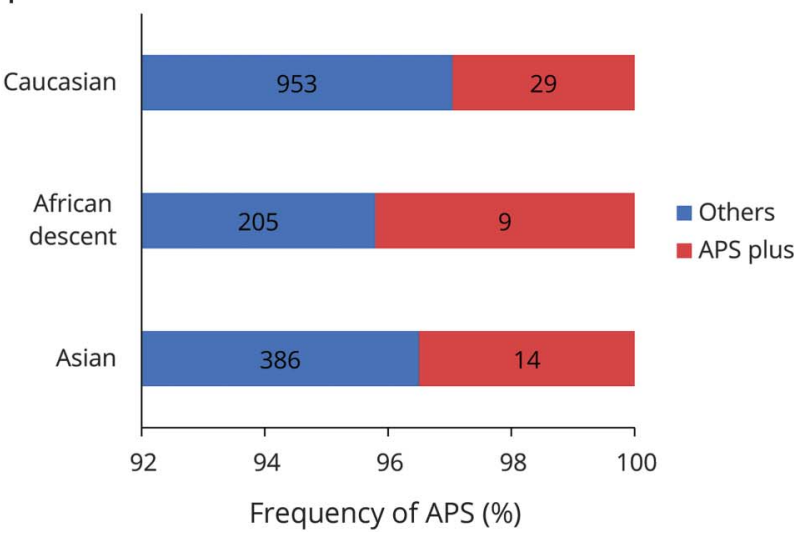

Frequency of APS, total number of attacks (A, B), either isolated APS (C, D) or along with other neurologic disorders (APS-plus: APS with optic neuritis and/or transverse myelitis and/or cerebral lesions [E, F]) during initial attack or subsequent relapses based on ethnicity in a multicenter international NMOSD database from 3 different countries (Japan [69 Asian descent patients], United Kingdom [118 Caucasian, 23 African descent, 16 Asian patients], and United States [122 Caucasian, 33 African descent, 19 Asian]). Note that details of APS on some patients included in this database may have been published in the context of single-center case series. APS = area postrema syndrome; NMOSD = neuromyelitis optica spectrum disorder.

(1), fatty liver (1), Clostridium difficile (1), and psychogenic (3).

\section{The phenotypic spectrum of APS includes intractable nausea, vomiting, and/or hiccups and is broader than previously recognized}

Seventy-three patients (73\%) had a single APS attack during their NMOSD course. Twenty-seven (27\%) had multiple APS attacks with a median APS attack frequency of 2 (1-9) (table 2). All 3 symptoms (nausea, vomiting, and hiccups $[\mathrm{NVH}])$ were present in 68 of 157 attacks (43\%, 45 patients), 2 symptoms ( $\mathrm{NV}[38], \mathrm{NH}[8], \mathrm{VH}[4])$ in 50 attacks (32\%, 32 patients), and a single symptom ( $\mathrm{N}$ [14], $\mathrm{H}[22], \mathrm{V}[3])$ in 39 (25\%, 23 patients).

\section{Nausea}

Nausea was acute in 73 attacks (57\%). Daily duration of nausea episodes was $\leq 6$ hours in 45 (35\%) and $>6$ hours in 83 (65\%). 
Table 2 APS phenotype of 157 attacks in 100 AQP4-IgG-positive patients with neuromyelitis optica spectrum disorder

$$
\text { APS symptoms in } 157 \text { attacks }
$$

\begin{tabular}{llll} 
& Nausea (N), 83 patients & Vomiting (V), ${ }^{\text {a } 75 \text { patients }}$ & $\begin{array}{l}\text { Hiccups (H) }{ }^{\mathbf{b}} \\
\mathbf{6 4} \text { patients }\end{array}$ \\
\hline No. (\%) with APS symptoms & $127(81)$ & $113(72)$ & $102(65)$ \\
\hline No. (\%) in isolation & $14(9)$ & $3(2)$ & $22(14)$ \\
\hline
\end{tabular}

No. (\%) with 1 other

NV 38 (24), NH 8 (5), VH 4 (3)

No. (\%) with all 3

$68(43)$

\begin{tabular}{|c|c|c|c|}
\hline Median duration, d (range) & $14(2-365)$ & $10(2-365)$ & $14(2-365)$ \\
\hline Continuous symptoms, n (\%) & $83(65)$ & $-^{\mathrm{c}}$ & $30(30)$ \\
\hline Episodic symptoms, $\mathbf{n}(\%)$ & $45(35)$ & $113(100)$ & $72(70)$ \\
\hline Episodes/d (range) & $5(2-30)$ & $5 \mathrm{~V} / \mathrm{d}(2-40)$ & $10(2-50)$ \\
\hline Median duration of each episode, min (range) & $10(2-120)$ & $2(1-20)$ & $15(2-300)^{d}$ \\
\hline Awakened due to symptom onset & 35 & 36 & 46 \\
\hline Weight loss (>3 lbs in $3 \mathrm{~d}$ ), $n$ & \multicolumn{3}{|c|}{75} \\
\hline Median, Ibs (range) & & $17(3-70)$ & \\
\hline
\end{tabular}

Abbreviations: APS = area postrema syndrome; AQP4-IgG = aquaporin-4-immunoglobulin G.

Twenty-seven patients reported migraine-like headaches in association with APS symptoms, characterized by severe throbbing headache, over the temples or back of the head with radiation to frontal regions that failed to respond to standard analgesia; 23 denied prior migraines or other headache history. Cardiac arrhythmias and fluctuations in blood pressure were reported in 16. Secondary amenorrhea was reported by 6 women during the course of the APS attack, 4 (2-10) months. Rare symptoms such as purposeful yawning unrelated to sleep or fatigue were reported in 2 patients, and generalized itching in another 2. a Of 100 patients, 91 of whom were women, 45 were Caucasian, 37 African American, 8 Asian, 7 Hispanic, and 3 others.

${ }^{\mathrm{b}}$ In no patient was hiccups triggered by food or drink. Hiccups improved in $15 \%$ of patients when supine.

c All vomiting attacks were episodic, even if it continued all day. Food and drink triggered vomiting in 9 patients. Vomiting was described as projectile by 8 patients.

${ }^{\mathrm{d}}$ Median interval between episodes was 10 minutes (1-480 minutes).

Figure 2 MRI abnormalities in AQP4-IgG-seropositive neuromyelitis optica spectrum disorder patients with APS

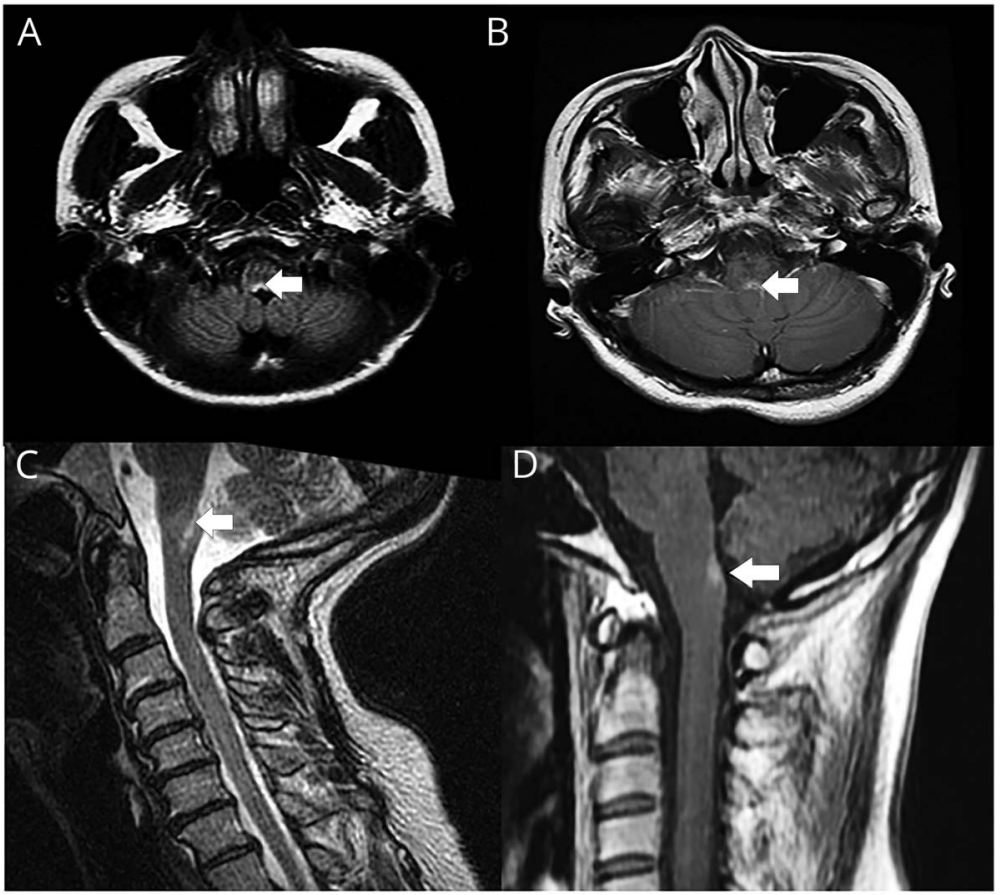

MRI head demonstrates a hyperintense lesion in the area postrema adjacent to the 4th ventricle on axial fluid-attenuated inversion recovery (A, arrow) in a patient with APS. In a separate patient, an enhancing lesion is noted the same region on axial T1-weighted head MRI postgadolinium administration ( $\mathrm{B}$, arrow). In a third patient a T2-hyperintense lesion in the area postrema is best seen on sagittal T2 weighted MRI of the cervical spine MRI (C, arrow). In a fourth patient, enhancement in the area postrema is demonstrated on sagittal T1-weighted cervical spine MRI post gadolinium (D, arrow). APS = area postrema syndrome; AQP4-lgG = aquaporin-4-immunoglobulin G. 


\section{Vomiting}

All vomiting attacks were episodic, even when persistent. The number of vomiting episodes in APS attacks was $\leq 4 / \mathrm{d}$ in 46 (41\%), 5-7 in 43 (38\%), and $\geq 8$ in 24 (21\%). Food and drink triggered vomiting only in 9 patients. Vomiting was described as projectile by 8 patients.

\section{Hiccups}

Onset was acute in 55 attacks (54\%). Daily duration of hiccups in APS attacks was $\leq 3$ hours in 31 (30\%), 4-12 hours in $27(27 \%)$, and $>12$ hours in $44(43 \%)$. Hiccups were reported as constant ("nonstop" for $>24$ hours) in 31 (30\%) APS attacks. While episodic in the remainder, the median interval between episodes was 10 minutes (1-480 minutes). In no patient was hiccups triggered by food or drink. Hiccups improved in $15 \%$ of patients when supine.

\section{Hospitalization is common, and symptomatic therapies are generally ineffective}

Because of symptom severity, 78 patients (78\%) required frequent emergency room visits for APS attacks. One hundred four attacks were treated with symptomatic treatments (i.e., antiemetics: Primperan [metoclopramide], ondansetron, promethazine) or IV rehydration only; partial improvement was reported in only 11 of these attacks (11\%) in the emergency room. APS symptoms led to hospitalization in 59 patients. For many patients, the symptoms would fluctuate over months and in some stopped spontaneously.

\section{Immunotherapy resulted in rapid cessation of symptoms in nearly $90 \%$ of patients treated}

Eighty-four APS attacks (in 51 patients) were treated with IV methylprednisolone (69 attacks) or plasmapheresis (15 attacks): $88 \%$ of APS attacks ceased within 2 days. In 10, symptoms partially improved, but eventually resolved with maintenance immunotherapy (azathioprine, mycophenolate mofetil, or rituximab).

\section{APS diagnostic criteria will assist in clinical diagnosis, attack identification, and classification for NMOSD clinical trials}

The criteria outlined in table 3 require AQP4-IgG seropositivity and acute or subacute onset of intractable nausea, vomiting, or hiccups. Single or combined symptoms are allowed. Symptoms must persist for at least 48 hours. Lack of response to symptomatic therapies may be a clue to APS. APS may only be diagnosed earlier than 48 hours if brain MRI reveals a new focus of signal abnormality in the AP given the broad differential diagnosis and the rarity of APS as a cause of vomiting and hiccups in the general population. APS can only be diagnosed after exclusion of other etiologies.

\section{Repurposing the PUQE scale for use in APS}

Nausea and vomiting severity was based on duration of nausea and frequency of vomiting episodes in accordance with validated PUQE criteria (tables 1 and 4). Using hiccups phenotype data, a separate hiccups section based on duration of symptoms was added (table 4). The severity of each symptom
Table 3 Area postrema syndrome criteria in AQP4IgG-seropositive neuromyelitis optica spectrum disorder

1. Acute or subacute NVH (single or combined symptoms), episodic or constant

2. Persistent for $\geq 48 \mathrm{~h},{ }^{a}$ with lack of complete resolution after symptomatic ${ }^{b}$ therapy

3. Exclusion of other etiology ${ }^{c}$

Abbreviations: AQP4-IgG = aquaporin-4-immunoglobulin G; NVH = nausea, vomiting, and hiccups.

For patients fulfilling criteria 1 to 3 , it is strongly recommended to test for AQP4-IgG if unknown.

a Shorter duration ( $<24$ hours) may be considered if MRI shows new area postrema involvement (figure 1, B).

${ }^{b}$ IV fluid, antiemetics, hiccups treatments.

'Metabolic (e.g., hyponatremia, liver dysfunction, renal dysfunction), gastrointestinal, biochemical, CNS structural lesions (e.g., tumor, stroke), mediastinal lesions, classic migraine, or psychiatric eating disorders.

type (nausea, vomiting, or hiccups) is graded on a 0 to 3 scale. An overall severity, defined as mild, moderate, or severe, is based on combinations of symptom grades. For example, a patient with hiccups lasting 3 hours or less and less than 4 episodes of vomiting in a 24-hour period would be considered "mild." In contrast, constant hiccups or nausea or more than 7 vomits per day would be considered "severe." In 100 US patients with NMOSD who had 157 APS attacks, 30 (19\%) were mild, 37 (24\%) were moderate, and 90 (57\%) were severe.

\section{Validation cohort}

Among the 25 NMOSD patients with APS, the majority, 20 (80\%), fulfilled criteria for severe APS; only 2 (8\%) were classified as mild and $3(12 \%)$ as moderate at APS attack nadir. Seventeen patients received symptomatic therapies: 12 (75\%) experienced no benefit; 4 (25\%) reported partial improvements, as was observed in the 100-patient cohort, and the overall APS severity grade dropped from severe to moderate in 3 and moderate to mild in 1 (figure 2). Eighteen patients received immunotherapy (IV methylprednisolone [12], plasma exchange [6]), 17 patients (95\%) experienced complete resolution of APS symptoms and their score returned to zero, and the other patient improved from severe to mild (figure 3). APS was isolated in $8(32 \%)$, and preceded typical NMOSD attacks in 17 patients (68\%): 11 (44\%) TM, $4(16 \%) \mathrm{ON}$, and 2 (8\%) brainstem.

\section{Discussion}

This multicenter, international, collaborative study provides new clinical and diagnostic insights into APS in AQP4IgG-positive NMOSD. APS is commonly encountered in AQP4-IgG-positive NMOSD across different ethnicities and continents, either in isolation or accompanying other symptoms (mostly ON or TM), at onset or during the course of the NMOSD illness. The phenotypic spectrum across 100 patients with 157 APS attacks is described. These 
Table 4 Area Postrema Severity Scale

\begin{tabular}{|c|c|}
\hline Over past $24 \mathrm{~h}$ & $\begin{array}{l}\% \text { of } 157 \text { APS } \\
\text { attacks in } \\
100 \text { patients }\end{array}$ \\
\hline \multicolumn{2}{|l|}{ Nausea grades } \\
\hline $0:$ None & 19 \\
\hline I: Total duration ${ }^{\mathrm{b}} \leq 3 \mathrm{~h}$ & 15 \\
\hline II: Total duration 3-6 h & 13 \\
\hline III: > $\mathrm{h}$ or constant & 53 \\
\hline \multicolumn{2}{|l|}{ Vomiting grades } \\
\hline 0: None & 28 \\
\hline I: $\leq 4$ episodes/d & 29 \\
\hline II: 5-7 episodes/d & 28 \\
\hline III: $\geq 8$ episodes/d ${ }^{c}$ & 15 \\
\hline \multicolumn{2}{|l|}{ Hiccups grade } \\
\hline 0: None & 35 \\
\hline I: Total duration ${ }^{b} \leq 3 \mathrm{~h} / \mathrm{d}$ & 20 \\
\hline II: Total duration 3-12 h/d & 17 \\
\hline III: $>12 \mathrm{~h}$ or constant ${ }^{\mathrm{c}}$ & 28 \\
\hline \multicolumn{2}{|l|}{ APSS } \\
\hline Normal: grade 0 & 0 \\
\hline Mild: 1 or 2 grade I & 19 \\
\hline Moderate: 1 or 2 grade II, or 3 grade I & 24 \\
\hline Severe: $\geq 1$ grade III, or 3 grade II & 57 \\
\hline
\end{tabular}

Abbreviations: APS = area postrema syndrome; APSS = Area Postrema Severity Scale.

aPSS measured at APS nadir.

${ }^{\mathrm{b}}$ May be episodic.

'Or experienced dehydration, electrolyte imbalance, or life-threatening condition.

observations facilitated proposal of diagnostic criteria for APS in NMOSD. Based on these phenotypic data, we propose an APS severity scale based on a previously validated severity scale for hyperemesis gravidarum (HG).

APS was included as a core clinical criterion of NMOSD in the revised 2015 NMOSD diagnostic guidelines and as 1 of 3 characteristic clinical manifestations, of which at least one was required in patients seronegative for AQP4-IgG. ${ }^{17}$ However, lack of specificity of these symptoms and absence of a rigorous definition leads to substantial subjectivity in its application by the treating physician, which may be problematic when comparing attack outcomes in clinical trials, of which there are currently 3 ongoing (MEDI-551 [anti-CD19], SA 237 [antiIL-6 receptor], eculizumab [anti-C5]). ${ }^{18,19}$

Given the high frequency of APS attacks in NMOSD and the increasing numbers of clinical trials aimed at reducing attack frequency (and severity), accurate diagnosis, especially of isolated APS, is important. Based on the APS phenotype reported here and defined through telephone questionnaire of prior APS attacks, diagnostic criteria for APS were developed. To enhance specificity, 48 hours was the minimum duration of symptoms allowed to make an APS diagnosis after exclusion of other etiologies and in the absence of a new MRI lesion in the AP. Although AP lesions are not specific for NMOSD, presence of intractable nausea, vomiting, and hiccups is highly specific for AQP4-IgG-seropositive NMOSD, which emphasizes the importance of clinical rather than MRI features of APS. ${ }^{20}$ Although in a clinical trial setting in which patients are encouraged to report symptoms as soon as they occur, we propose that diagnosis of INVH as an APS earlier than 48 hours of onset is possible only when a dorsal medulla lesion is detected on MRI.

Given that $75 \%$ of patients reported weight loss, up to 70 pounds, we suspect that anorexia was a contributing factor in our cohort. Lesions in the AP in animal models can induce hypophagia and weight loss without alteration in intestinal transit or gastric retention. ${ }^{21}$

In support of the specificity of this syndrome as a manifestation of NMOSD, AQP4-IgG was not detected in 435 patients (enrolled in the NIH-funded Gastroparesis Clinical Research Consortium repository) who presented with idiopathic nausea or vomiting. ${ }^{16}$ The 435 patients enrolled in the Gastroparesis Clinical Research Consortium Registry cohort included 318 patients with gastroparesis, of whom 59\% had vomiting or nausea as their predominant symptom that prompted gastroenterologic evaluation. Their Patient Assessment of Upper Gastrointestinal Disorders-Symptoms Severity Index (PAGI-SYM) scores (scale $0-5$, where $0=$ none and $5=$ very severe) were $3.5 \pm 1.2$ for nausea and $2.2 \pm$ 1.9 for vomiting. The frequency of vomiting or nausea as the predominant symptom that prompted gastroenterologic evaluation in those lacking gastroparesis was $60 \%$ for nausea and vomiting. Their PAGI-SYM scores were $3.4 \pm 1.3$ for nausea and $1.9 \pm 1.9$ for vomiting. ${ }^{16}$

However, recognizing the lack of specificity of nausea and vomiting, in the absence of detectable AQP4-IgG or for symptoms lasting less than 48 hours, the criteria for an APS attack requires a new $\mathrm{T} 2$ or enhancing MRI lesion in the AP for confirmation.

Most APS attacks (58\% of the Mayo-only cohort and $68 \%$ of the international validation cohort) preceded ( $<30$ days, thus considered the same attack) inflammatory involvement of the optic nerves or spinal cord, making APS an important warning sign. Early treatment of APS with steroids or plasmapheresis (or other acute attack immunotherapies) may not only reduce severity of APS attacks but may also prevent accompanying disabling attacks of ON or TM. Therefore, evaluating the severity may be important in NMOSD clinical trials. Sensitivity of outcome measures is particularly important for 


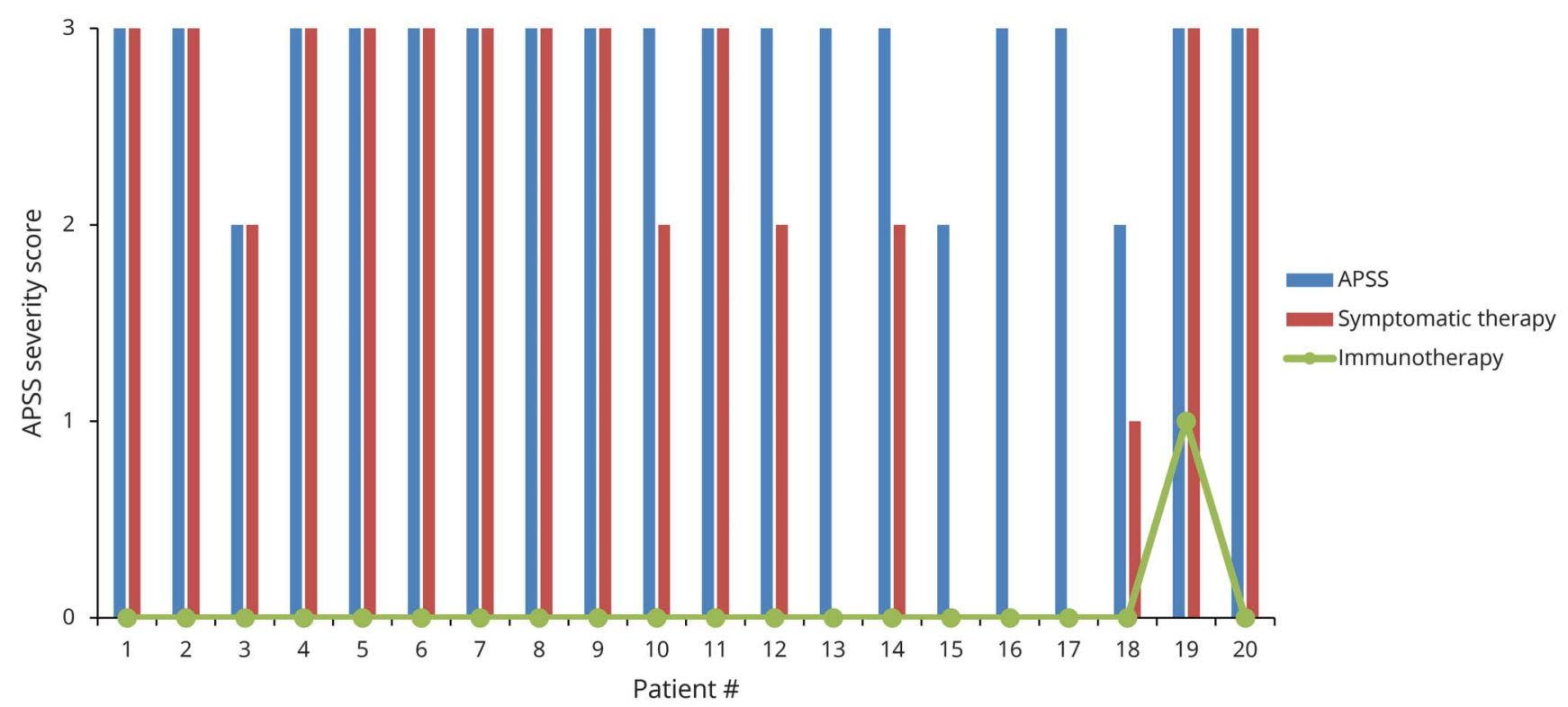

Change in APSS scores after symptomatic and immunosuppressant therapies for 20 patients with both nadir and posttherapy scores. APSS = Area Postrema Severity Scale.

NMOSD studies that typically are "time to first event" studies with each patient contributing information only from a single attack before removal from the blinded phase of a clinical trial.

Up to $80 \%$ of all pregnant women report nausea and vomiting, and in some, this is associated with a reduced quality of life. ${ }^{22}$ A more serious condition, $\mathrm{HG}$, occurs in approximately $1 \%$ of pregnant women. HG bears some similarities to APS in NMOSD, and is defined as vomiting occurring before the 20th week of gestation of such severity as to require the patient's admission to hospital. The pregnancy-specific questionnaire PUQE score was developed to assess the severity of emesis (nausea and vomiting) in pregnancy. ${ }^{23}$ It contains 3 questions regarding the time span of nausea, vomiting, and retching, respectively. The questionnaire has been modified to encompass 24 hours as well as the entire first trimester (table 1). ${ }^{24}$ PUQE score has been validated to correlate with inability to take iron supplementation in pregnancy, risk of hospitalization due to $\mathrm{HG}$ or severe nausea and vomiting in pregnancy, increased health care costs attributable to nausea and vomiting in pregnancy, and reduced well-being/quality of life. ${ }^{25}$ The PUQE questionnaire has also been used in several studies assessing the effect of antiemetic treatments for emesis and hyperemesis. ${ }^{26,27}$ The severity scale for APS proposed herein is based on the nausea and vomiting questionnaire sections of the PUQE criteria. Intractable hiccups severity scale, based on duration of symptoms, is an additional component in the APS scoring system. In the multicenter validation cohort, the severity grades were similar to those in the 100-patient cohort. The APS severity scale detected improvements with both symptomatic therapy and immunotherapy, demonstrating the potential utility in drug trials. This study was restricted to patients with the immunopathologically distinct entity AQP4-IgG NMOSD. It remains unclear whether these data are applicable to APS that have been observed in patients with myelin oligodendrocyte glycoprotein autoimmunity. ${ }^{1,28}$ The applicability to seronegative diseases remains unknown.

\section{Author contributions}

Conception and design of the study: S.J.P., B.W., E.S. Acquisition and analysis of data: all authors. Drafting a significant portion of the manuscript or figures: S.J.P., E.S., B.M.G., D.D.

\section{Acknowledgment}

The authors thank Mrs. Mary Curtis for her secretarial work.

\section{Study funding}

This study was funded in part by the NIH (RO1 NS06582901), Guthy-Jackson Charitable Foundation, the Center for MS and Autoimmune Neurology, Mayo Clinic, and Alexion Pharmaceuticals. The Article Processing Charge was funded by Mayo Clinic.

\section{Disclosure}

E. Shosha and D. Dubey report no disclosures relevant to the manuscript. J. Palace has received support for scientific meetings and honorariums for advisory work from Merck Serono, ABIDE, Biogen Idec, Novartis, Alexion, MedImmune, Teva, Chugai Pharmaceutical, and Bayer Schering, and unrestricted grants from Merck Serono, Novartis, Biogen Idec, and Bayer Schering; grants from the MS Society; GMSI, 
NIHR, and Guthy-Jackson Foundation for research studies. The Oxford NMO service is supported by highly specialized commissioning. I. Nakashima reports no disclosures relevant to the manuscript. A. Jacob has received honoraria for speaking on NMOSD from Biogen, Chugai, Shire, and Terumo BCT Pharmaceuticals and has received funding for investigator-sponsored studies from Shire, Alexion, and Biogen. He has served on the clinical trial advisory boards of Shire and Chugai Pharmaceutical. K. Fujihara serves on scientific advisory boards for Bayer Schering Pharma, Biogen Idec, Mitsubishi Tanabe Pharma Corporation, Novartis Pharma, Chugai Pharmaceutical, Ono Pharmaceutical, Nihon Pharmaceutical, Merck Serono, Alexion Pharmaceuticals, MedImmune, and Medical Review; has received funding for travel and speaker honoraria from Bayer Schering Pharma, Biogen Idec, Eisai Inc., Mitsubishi Tanabe Pharma Corporation, Novartis Pharma, Astellas Pharma Inc., Takeda Pharmaceutical Company Limited, Asahi Kasei Medical Co., DaiichiSankyo, and Nihon Pharmaceutical; serves as an editorial board member of Clinical and Experimental Neuroimmunology (2009 to present) and an advisory board member of Sri Lanka Journal of Neurology; has received research support from Bayer Schering Pharma, Biogen Idec Japan, Asahi Kasei Medical, The Chemo-Sero-Therapeutic Research Institute, Teva Pharmaceutical, Mitsubishi Tanabe Pharma, Teijin Pharma, Chugai Pharmaceutical, Ono Pharmaceutical, Nihon Pharmaceutical, and Genzyme Japan; is funded by Grants-in-Aid for Scientific Research from the Ministry of Education, Culture, Sports, Science and Technology of Japan (22229008, 2010-2015; 26293205, 2014-2016) and by Grants-in-Aid for Scientific Research from the Ministry of Health, Welfare and Labor of Japan (2010 to present). T. Takahashi has received research support from Cosmic Corporation. D. Whittam reports no disclosures relevant to the manuscript. M. Leite is involved in AQP4 testing; is supported by the National Health Service National Specialised Commissioning Group for Neuromyelitis Optica and by the National Institute for Health Research Oxford Biomedical Research Centre; and has received speaking honoraria from Biogen Idec and a travel grant from Novartis. T. Misu and T. Yoshiki report no disclosures relevant to the manuscript. S. Messina has received honoraria for scientific lectures from Biogen, Almirall, travel payment from Novartis, Biogen Idec, Genzyme, Almirall Bayer Schering, Merck, Serono, and Teva, and served on the scientific advisory board for Biogen. L. Elsone, M. Majed, E. Flanagan, A. Gadoth, C. Huebert, and J. Sagen report no disclosures relevant to the manuscript. B. Greenberg has received research support from NIH, PCORI, NMSS, GuthyJackson Charitable Foundation, Chugai, MedImmune, MedDay, and Genentech and serves as a consultant to Alexion and Novartis. M. Levy receives research support from NIH, Guthy-Jackson Charitable Foundation, ViroPharma, Acorda, Sanofi, Neuralstem, and Genentech, and serves as a consultant for Chugai Pharmaceuticals, GlaxoSmithKline, and MedImmune. A. Banerjee reports no disclosures relevant to the manuscript. B. Weinshenker receives royalties from RSR Ltd., Oxford University, Hospices Civil de Lyon, and MVZ
Labor PD Dr. Volkmann und Kollegen GbR for a patent of NMO-IgG as a diagnostic test for NMO and related disorders. He serves as a member of an adjudication committee for clinical trials in NMO being conducted by MedImmune and Alexion pharmaceutical companies. He is a consultant for Caladrius Biosciences and Brainstorm Therapeutics regarding a clinical trial for NMO. S. Pittock and Mayo Clinic have financial interest in patents (12/678,350 filed 2010 and 12/ 573,942 filed 2008) that relate to functional AQP4/NMOIgG assays and NMO-IgG as a cancer marker. He has served as a consultant to Alexion Pharmaceuticals and MedImmune. He has received research funding from Alexion, MedImmune, and Grifols. Go to Neurology.org/N for full disclosures.

\section{Publication history}

Received by Neurology April 2, 2018. Accepted in final form July 18, 2018.

\section{References}

1. Pittock SJ, Lucchinetti CF. Neuromyelitis optica and the evolving spectrum of autoimmune aquaporin-4 channelopathies: a decade later. Ann NY Acad Sci 2016;1366: 20-39.

2. Lennon VA, Wingerchuk DM, Kryzer TJ, et al. A serum autoantibody marker of neuromyelitis optica: distinction from multiple sclerosis. Lancet 2004;364: 2106-2112.

3. Hinson SR, Pittock SJ, Lucchinetti CF, et al. Pathogenic potential of IgG binding to water channel extracellular domain in neuromyelitis optica. Neurology 2007;69: 2221-2231.

4. Amiry-Moghaddam M, Ottersen OP. The molecular basis of water transport in the brain. Nat Rev Neurosci 2003;4:991-1001.

5. Pittock SJ, Weinshenker BG, Lucchinetti CF, Wingerchuk DM, Corboy JR, Lennon VA. Neuromyelitis optica brain lesions localized at sites of high aquaporin 4 expression. Arch Neurol 2006;63:964-968.

6. Duvernoy HM, Risold PY. The circumventricular organs: an atlas of comparative anatomy and vascularization. Brain Res Rev 2007;56:119-147.

7. Gross PM. Morphology and physiology of capillary systems in subregions of the subfornical organ and area postrema. Can J Physiol Pharmacol 1991;69: 1010-1025.

8. Price CJ, Hoyda TD, Ferguson AV. The area postrema: a brain monitor and integrator of systemic autonomic state. Neuroscientist 2008;14:182-194.

9. Misu T, Fujihara K, Nakashima I, Sato S, Itoyama Y. Intractable hiccup and nausea with periaqueductal lesions in neuromyelitis optica. Neurology 2005;65: 1479-1482.

10. Apiwattanakul M, Popescu BF, Matiello M, et al. Intractable vomiting as the initial presentation of neuromyelitis optica. Ann Neurol 2010;68:757-761.

11. Popescu BF, Lennon VA, Parisi JE, et al. Neuromyelitis optica unique area postrema lesions: nausea, vomiting, and pathogenic implications. Neurology 2011;76: 1229-1237.

12. Lindstrom PA, Brizzee KR. Relief of intractable vomiting from surgical lesions in the area postrema. J Neurosurg 1962;19:228-236.

13. Hornby PJ. Central neurocircuitry associated with emesis. Am J Med 2001;111(suppl $8 \mathrm{~A}): 106 \mathrm{~S}-112 \mathrm{~S}$

14. Sato D, Fujihara K. Atypical presentations of neuromyelitis optica. Arq Neuropsiquiatr 2011;69:824-828.

15. Takahashi T, Miyazawa I, Misu T, et al. Intractable hiccup and nausea in neuromyelitis optica with anti-aquaporin-4 antibody: a herald of acute exacerbations. J Neurol Neurosurg Psychiatry 2008;79:1075-1078.

16. Iorio R, Lucchinetti CF, Lennon VA, et al. Intractable nausea and vomiting from autoantibodies against a brain water channel. Clin Gastroenterol Hepatol 2013;11: 240-245.

17. Wingerchuk DM, Banwell B, Bennett JL, et al. International consensus diagnostic criteria for neuromyelitis optica spectrum disorders. Neurology 2015;85:177-189.

18. Pittock SJ, Lennon VA, McKeon A, et al. Eculizumab in AQP4-IgG-positive relapsing neuromyelitis optica spectrum disorders: an open-label pilot study. Lancet Neurol $2013 ; 12: 554-602$.

19. Cree BA, Bennett JL, Sheehan M, et al. Placebo-controlled study in neuromyelitis optica: ethical and design considerations. Mult Scler 2016;22:862-872.

20. Dubey D, Pittock SJ, Krecke KN, Flanagan EP. Association of extension of cervical cord lesion and area postrema syndrome with neuromyelitis optica spectrum disorder. JAMA Neurol 2017;74:359-361.

21. Hyde TM, Miselis RR. Effects of area postrema/caudal medial nucleus of solitary tract lesions on food intake and body weight. Am J Physiol 1983;244: R577-R587.

22. Chortatos A, Haugen M, Iversen PO, Vikanes A, Magnus P, Veierod MB. Nausea and vomiting in pregnancy: associations with maternal gestational diet and 
lifestyle factors in the Norwegian Mother and Child Cohort Study. BJOG 2013; 120:1642-1653.

23. Koren G, Boskovic R, Hard M, Maltepe C, Navioz Y, Einarson A. Motherisk-PUQE (Pregnancy-Unique Quantification of Emesis and Nausea) scoring system for nausea and vomiting of pregnancy. Am J Obstet Gynecol 2002;186:S228-S231.

24. Lacasse A, Rey E, Ferreira E, Morin C, Berard A. Validity of a modified Pregnancy-Unique Quantification of Emesis and Nausea (PUQE) scoring index to assess severity of nausea and vomiting of pregnancy. Am J Obstet Gynecol 2008;198:71.e1-71.e7.

25. Koren G, Piwko C, Ahn E, et al. Validation studies of the Pregnancy UniqueQuantification of Emesis (PUQE) scores. J Obstet Gynaecol 2005;25:241-244.
26. Birkeland E, Stokke G, Tangvik RJ, et al. Norwegian PUQE (Pregnancy-Unique Quantification of Emesis and Nausea) identifies patients with hyperemesis gravidarum and poor nutritional intake: a prospective cohort validation study. PLoS One 2015; 10:e0119962.

27. Maltepe C, Koren G. The management of nausea and vomiting of pregnancy and hyperemesis gravidarum: a 2013 update. J Popul Ther Clin Pharmacol 2013;20: e184-e192.

28. Zamvil SS, Slavin AJ. Does MOG Ig-positive AQP4-seronegative opticospinal inflammatory disease justify a diagnosis of NMO spectrum disorder? Neurol Neuroimmunol Neuroinflamm 2015;2:e62. 


\section{Neurology}

\section{Area postrema syndrome: Frequency, criteria, and severity in AQP4-IgG-positive NMOSD}

Eslam Shosha, Divyanshu Dubey, Jacqueline Palace, et al.

Neurology 2018;91;e1642-e1651 Published Online before print September 26, 2018

DOI 10.1212/WNL.0000000000006392

This information is current as of September 26, 2018

\author{
Updated Information \& \\ Services \\ Supplementary Material \\ References \\ Citations \\ Permissions \& Licensing \\ Reprints
}

including high resolution figures, can be found at: http://n.neurology.org/content/91/17/e1642.full

Supplementary material can be found at: http://n.neurology.org/content/suppl/2020/03/04/WNL.0000000000006 392.DC1

This article cites 28 articles, 4 of which you can access for free at: http://n.neurology.org/content/91/17/e1642.full\#ref-list-1

This article has been cited by 2 HighWire-hosted articles: http://n.neurology.org/content/91/17/e1642.full\#\#otherarticles

Information about reproducing this article in parts (figures,tables) or in its entirety can be found online at:

http://www.neurology.org/about/about_the_journal\#permissions

Information about ordering reprints can be found online:

http://n.neurology.org/subscribers/advertise

Neurology ${ }^{\circledR}$ is the official journal of the American Academy of Neurology. Published continuously since 1951 , it is now a weekly with 48 issues per year. Copyright Copyright (C) 2018 The Author(s). Published by Wolters Kluwer Health, Inc. on behalf of the American Academy of Neurology.. All rights reserved. Print ISSN: 0028-3878. Online ISSN: 1526-632X.

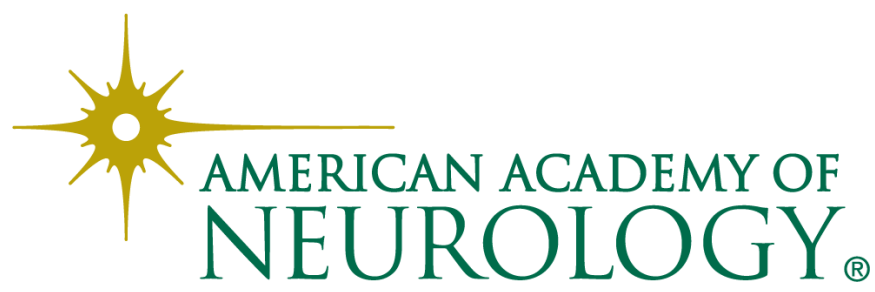

